\title{
Study on Remote Monitoring System of Subway Central Air-conditioning Systems Based on Internet of Things
}

\author{
Lingyun Wang ${ }^{1, a}$, Wei Zhang ${ }^{2, b}$, Linghong $\mathrm{Xu}^{3, \mathrm{c}}$ and Peng $\mathrm{Ni}^{4, \mathrm{~d}}$ \\ ${ }^{1}$ School of Electrical \& Electronic Engineering, Huazhong University of Science and Technology, \\ Wuhan, China \\ ${ }^{2,3,4}$ School of Environmental Science \& Engineering, Huazhong University of Science and \\ Technology, Wuhan, China \\ âwangly@hust.edu.cn, 'bhangweihust@qq.com, 'Ih.xu@hust.edu.cn, d1553638882@qq.com
}

\begin{abstract}
Keywords: Internet of Things, Subway central air conditioning, VPN technology, Remote monitor Abstract. This paper proposes a scheme for remote monitoring system of central air-conditioning systems of subways based on the Internet of Things technology after analyzing the characteristics of such technology and the function requirements of the system. The general structure of this scheme, which includes perception layer, network layer and application layer were investigated. This scheme integrates all air-conditioning systems of subway stations to achieve remote monitoring and management as well as conservation of related manpower and operating costs. This scheme applies the virtual local area network (VPN) technology in its network layer to improve the security, stability, and real-time performance of the remote monitoring system. A VPN tunnel is also be established between the mobile terminal and the monitoring center to enable staff members to monitor each central air-conditioning system by using a "pocket" or "mobile" terminal.
\end{abstract}

\section{Introduction}

The rapid urbanization of China has driven many people to relocate to cities, thereby increasing the pressure of urban traffic and encouraging the development of subways. Subway stations have important roles in subway systems. As an indispensable equipment of these stations, central air-conditioning systems provide passengers with a comfortable environment, but it also consumes a large amount of energy. Apart from train traction and traction power, central air-conditioning systems consume the largest amount of energy among all pieces of equipment available in subways. The central air-conditioning systems account for more than $50 \%$ of consumed energy in metro stations ${ }^{[1]}$. A large number of subway stations are dispersed across cities, with each city having at least 10 subway routes. Therefore, subway operation units must determine how to achieve unified monitoring and management of their stations, reduce their energy consumption, and save the costs of using central air-conditioning systems. The Internet of Things (IoT) has attracted widespread attention and is currently considered as the third wave of the information industry after computers, Internet, and mobile communication networks. This technology has been successfully applied in the fields of intelligent transportation, environmental monitoring, industrial control, and intelligent fire protection ${ }^{[2-3]}$, but its application in the field of urban subways has rarely been investigated ${ }^{[4-5]}$. Therefore, this paper proposes a scheme for the remote monitoring system of central air-conditioning systems in subways based on the IoT technology and investigate the characteristics of central air-conditioning systems in subways. The conclusion from this paper also contributes to similar projects as an valuable reference.

\section{Design of Remote Monitoring and Control Systems for Central Air-conditioning Systems of Subways}

Overall System Architecture. According to the functional requirements of the central air-conditioning remote monitoring system and the technical characteristics of IoT, this paper proposes a remote monitoring system for the central air-conditioning systems of subways. Fig. 1 presents the overall architecture of the remote monitoring system. The system is divided into three different layers: scene perception layer, network layer and application layer, and physically comprises one field data 
acquisition set, several industrial-strength wireless routers with the function of a virtual local area network (VPN), servers, and one monitoring platform. The central air-conditioning systems along a subway line can be integrated by this scheme to enable managers remotely monitoring the systems in each station through a client, browser, or mobile terminal anytime and anywhere. By improving the efficient management of air-conditioning systems, the proposed scheme can solve the difficulties in maintenance and performance well in cost reduction.

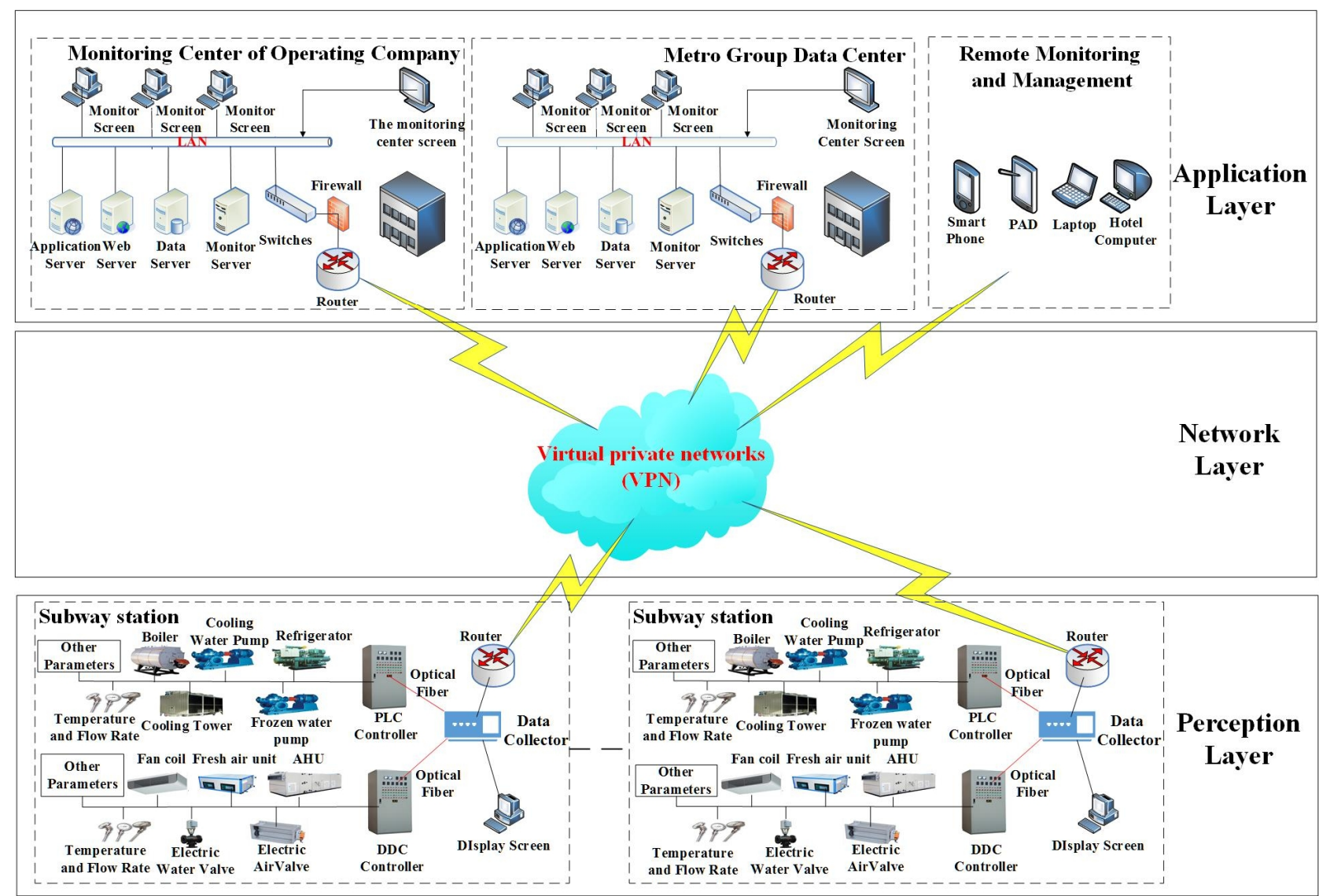

Fig.1. General structure of remote monitoring system for central air-conditioning systems in subways.

Design of Scene Perception Layer. The bottom part of Fig. 1 shows the perception layer, which is designed to collect data from the central air-conditioning systems of subway stations and to provide a human-computer interface that can help subway station managers monitor these systems. The building automation system is installed in $90 \%$ of subway stations in China to monitor and control their electromechanical equipment ${ }^{[6]}$. To fully utilize the existing resources and reduce capital investment, the field control layer applies the programmable logic controller (PLC) control system and the end point of the direct digital controller (DDC) control system, which are originally integrated in the central air-conditioning systems of subways in the proposed scheme. In this way, the remote monitoring system reads the parameters in machine room and air-conditioning system equipment directly from the original PLC and DDC control cabinets through an optical fiber.

As the most important component in this scheme, the PLC and DDC control systems are used to send all kinds of field signals to the control operation to control all field devices ${ }^{[7-9]}$. Therefore, choosing one satisfied controller is essential to ensure the reliability of field data acquisition and control. The input signals of the calculator room equipment include some analog signals (i.e., supply and return water temperature of chilled and cooling water, pressure, and flow) and digital signals (i.e., start and stop of refrigerating units, pumps, and cooling tower). Similarly, the input signals of the end equipment include some analog signals (i.e., the temperature of supply and return air, and supply air volume) and digital signals (i.e., start and stop of fan, electric air valve, and electric water valve).

Design of Network Layer. Subway stations can be divided into public areas and management/ equipment rooms. To make passengers feel comfortable and ensure the safe usage of management/ 
equipment rooms, the air-conditioning systems in public areas must function continuously during their operating times, and the air-conditioning systems of the management/equipment room must function continuously 24 hours a day ${ }^{[12]}$. The application layer can achieve data interaction and resource sharing with the scene perception layer through the network layer. Therefore, the stability and security of the network layer is highly required to guarantee the real-time transmission and normal operation of monitoring on the central air-conditioning systems under all weather conditions 24 hours a day.

As shown in Fig.2, the VPN technology is selected as the core in this study to establish the network layer in the system. Each subway station field end is connected to the Internet through the dual-redundancy network of ADSL and 4G, whereas the monitoring center of the subway operation unit is connected to the Internet through an optical fiber. To guarantee the security, stability, and real-time transmission capacity of the entire transmission network, the system sets up a VPN IPsec tunnel on the router, builds a virtual private network based on the public network between the field ends and monitoring center ${ }^{[13-15]}$, and places the subnet of both ends in LAN. At the same time, a VPN IPsec tunnel is built between the corporation information center and the monitoring center of the subway operation unit to inform the corporation about the operation conditions of each air-conditioning system and to share information resources. A VPN SSL tunnel is also established between the mobile terminal (i.e., mobile phone and tablet PC) and the monitoring center to enable the operation unit remotely monitoring the central air-conditioning system of each station. Therefore, the proposed system provides these units with a "pocket" or "mobile office."

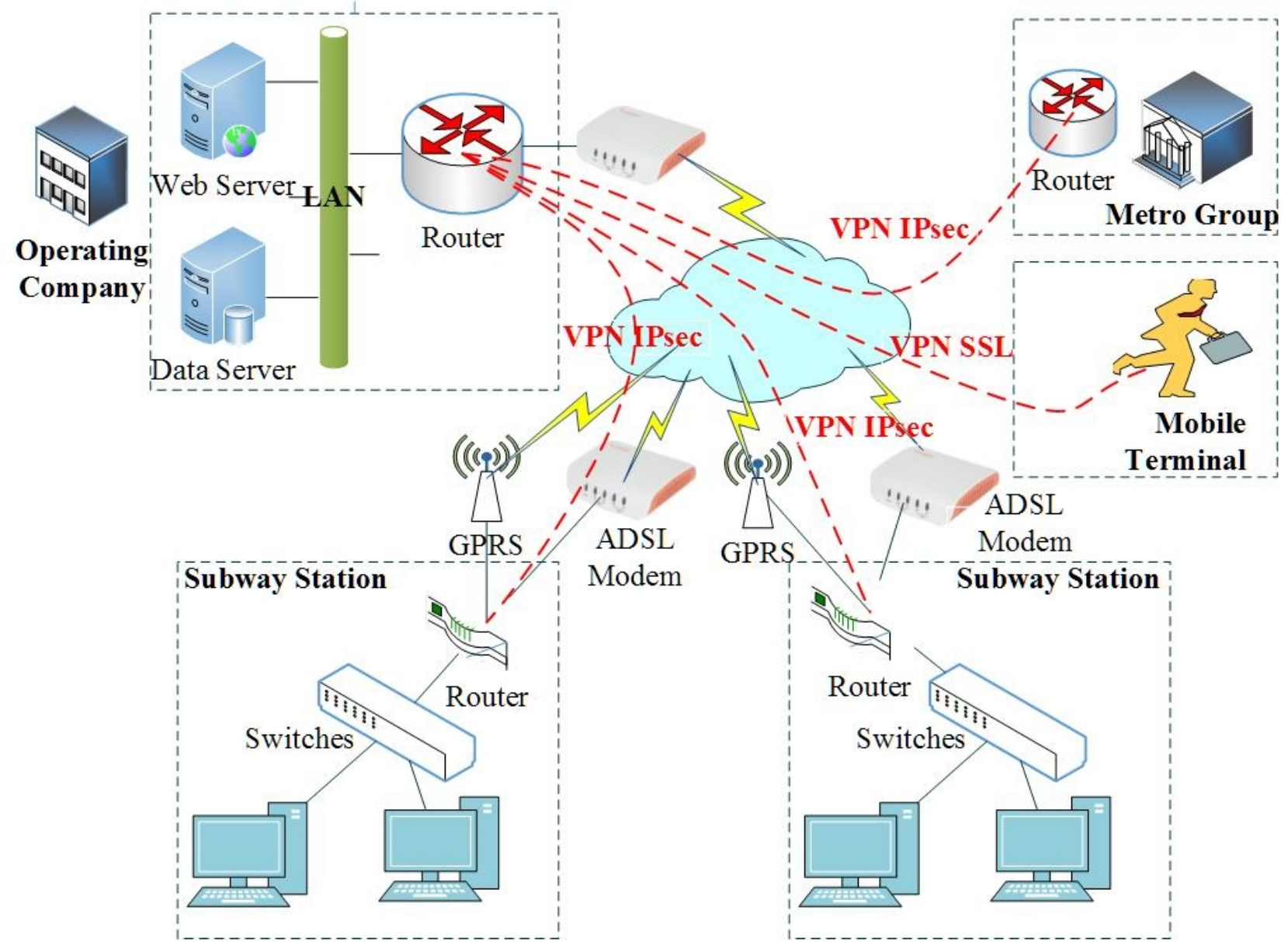

Fig.2. Remote monitoring and control systems for central air-conditioning systems of subways

Design of Application Layer. As shown in Fig.1, the application layer is divided into the monitoring center of the operation company, the data center of the metro group corporation, and the remote client. The monitoring center for the operation company and the data center for the metro group corporation consist of server and configuration software, while the remote client consists of a PC or mobile terminals that can connect to the Internet. The configuration software is placed at the core of the application layer. The operation condition data that are uploaded by the network layer can 
be displayed and stored through the configuration software to analyze the amount of energy that is being consumed by each air-conditioning system. Based on the equipment, season, climate, time, and other factors, all types of dispatching commands can be sent to the scene perception layer to control the air-conditioning equipment remotely. Therefore, the configuration software must be stable and reliable. The proposed scheme uses Indusoft Web Studio from Schneider, which can build a centralized monitoring system based on $\mathrm{B} / \mathrm{S}$ and $\mathrm{C} / \mathrm{S}$ architecture to allow managers to control the air-conditioning equipment remotely through a browser, client, or mobile terminal, thereby reducing energy consumption $^{[16]}$.

\section{Design of Data Stream}

Numerous subway stations are distributed widely across China, with each station containing plenty of real-time and historical data. Therefore, a clear data flow scheme must be proposed to guarantee that the remote monitoring system runs safely, stably, and efficiently. Fig.3 shows the data flow design of the remote monitoring system.

In the proposed scheme, the data platform of each subway station and operation center is built according to real-time database to ensure that the real-time data and historical data can function smoothly. The real-time database is the key component of data acquisition and echo for I/O equipment through the $\mathrm{I} / \mathrm{O}$ drivers in monitoring system ${ }^{[17]}$. The database constructs a two-way communication with process data and implement the real-time processing of the entire system, storage of historical data, and responding to data requests.

As shown in Fig.3, the equipment state parameters of the equipment room at the subway station (including water chiller, water pump, cooling tower, and other related equipment state parameters), the terminal equipment state parameters (including the end of air-conditioning units, fresh air handling units, and fan-coil state parameters), the environmental parameters, and the real-time data of the air-conditioning water system are collected and sent to the real-time database of the station through a protocol. Then, these data are synchronized with the real-time database through the TCP or OPC protocol. The real-time database of the monitoring center provides data services for other real-time or non-real-time systems through XML, OPC, TCP, FTP, or SMTP. The data collected by the field-end database of the station are stored into a relational database and are then synchronized with the central station relational database through OLEDB to provide historical data services for monitoring the center business platform. When some stations need to be remotely controlled and adjusted, the operation monitoring center can change the real-time database point value of the monitoring center, synchronize this value to the central station relational database through the TCP or OPC protocol, and then complete the control and regulation of the central air-conditioning system of each station. 


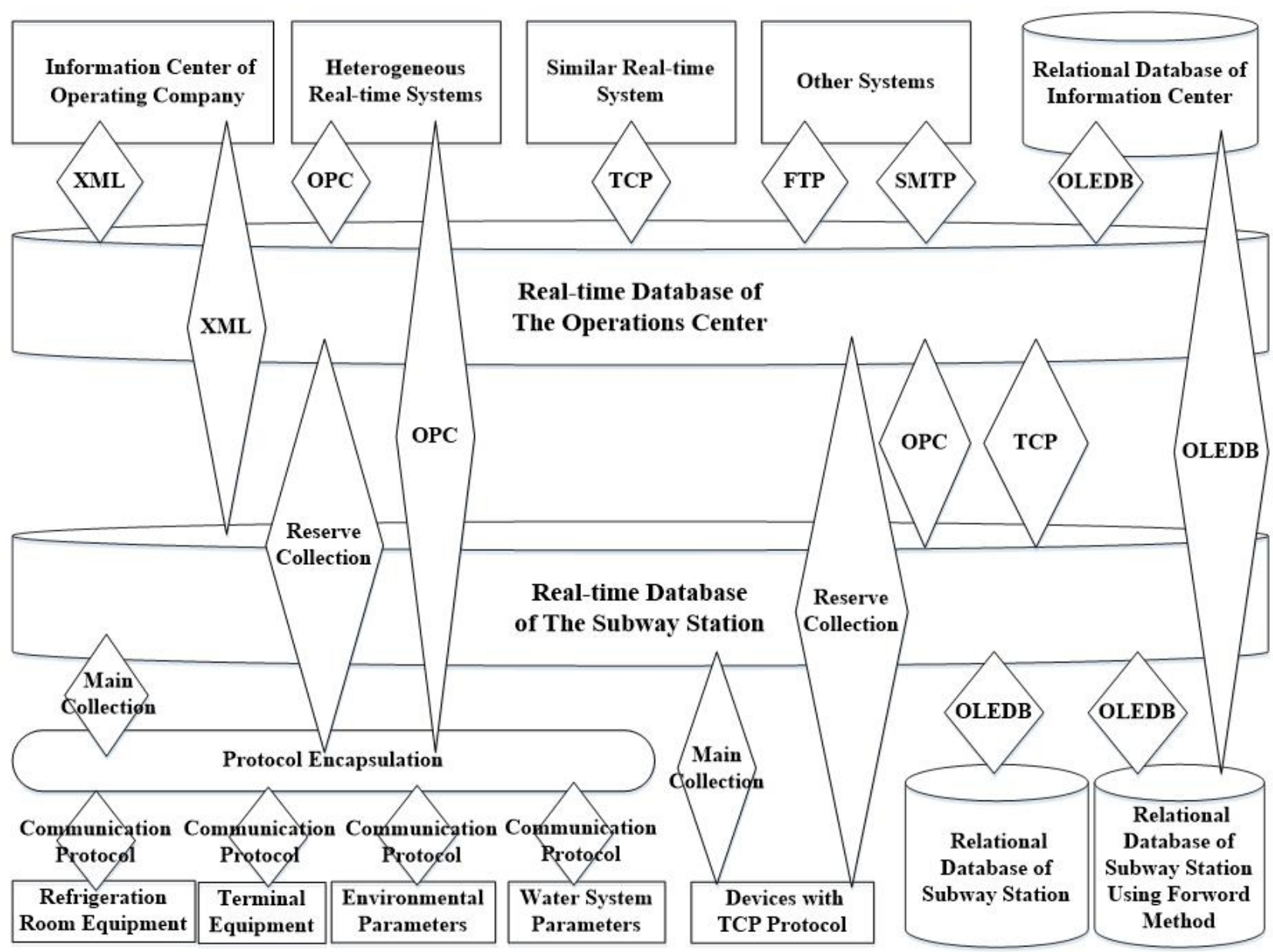

Fig.3. Data flow chart of remote monitoring and control systems for central air-conditioning systems of subways

\section{Conclusions}

By analyzing the characteristics of the IoT technology and the functional requirements of the central air-conditioning systems of subways, this paper proposes a central air-conditioning remote monitoring system and describes the general structure, scene perception layer design, network layer design, application layer design, and data flow design in detail. Apart from achieving a unified remote monitoring and management of the central air-conditioning systems of all subway stations, the proposed system improves the efficiency of these air-conditioning systems, decreases energy consumption, and reduces human power and operating costs. The network layer using VPN technology can greatly improve the security, stability, and real-time data collection of the system. The proposed scheme creates a "pocket" or "mobile" office that establishes a VPN tunnel between the mobile terminal (i.e., mobile phones and PAD hotel computers) and the monitoring center to help staff members monitor the central air-conditioning system of each subway station anytime and anywhere. Implementing this scheme has far-reaching significance in the monitoring and management of central air-conditioning systems.

\section{References}

[1] Xiaoling Ding. The Application and Research of Self-Adjusting Quantization Scale Factor Fuzzy Control in the Central Air Conditioning Water System [D]. Jinan: Shandong University, master's degree thesis, 2007. (In Chinese)

[2] Atzori L, Iera A, Morabito G. The Internet of Things: A survey [J]. Computer Networks the International Journal of Computer \& Telecommunications Networking, 2010, 54(15):2787-2805. 
[3] Borgia E. The Internet of Things vision: Key features, applications and open issues [J]. Computer Communications, 2014, 54:1-31.

[4] Jun Yuan. Design and realization of the subway station BAS simulation platform [D]. Beijing: Beijing University of Technology master's degree thesis, 2013. (In Chinese)

[5] Xianchen Peng. The Research of design and implementation in the building automatic system of metro [D]. Lanzhou: Lanzhou University of Technology master's degree thesis, 2013. (In Chinese)

[6] Xiaoming Jiang, Chunlin Tan. Energy-Saving Control of Metro Central Air-Conditioning [J]. Mechanical \& Electrical Engineering Technology, 2013, 42(07):30-33. (In Chinese)

[7] Price, B.A, Smith T F, Suby A A, et al. Calibration and uncertainty analysis of temperature sensors [HVAC DDC system] [C]// Sensors for Industry, 2001. Proceedings of the First ISA/IEEE Conference. IEEE, 2001:286-289.

[8] Alphonsus, E. R, Abdullah M O, A review on the applications of programmable logic controllers (PLCs) [J]. Renewable and Sustainable Energy Reviews 60 (2016) 1185-1205.

[9] Gang W. Design of Ice-Storage Air Conditioning Control System Based on PLC and Touching Screen [J]. Refrigeration \& Air Conditioning, 2009, 3:261-264.

[10]Peng Zhang, Huanlin He, Yanxia Peng. Research and implementation of the central air conditioning SCADA system based on Internet of Things [J]. Industrial Instrumentation \& Automation, 2014(06):69-73. (In Chinese)

[11]Peng Zhang, Pengju He. Prototype Architecture of the Medical Cyber Physical System based on Grid Computing[J]. The International Conference on Electrical Engineering and Automatic, 2010.

[12] Jie Xu. Energy-saving control of the Subway Station Central air conditioning system [D]. Hefei: Hefei University of Technology master's degree thesis, 2013. (In Chinese)

[13] Jinhao L, Chi D. Study on the application of VPN technology based on IPSec in the modern universities [J]. Beijing: 2011.

[14]Luo Z, You B, Wang J, et al. Application of VPN Technology in Multi-Campus Adult Education Platform[J]. Haikou: 2014.

[15] Xiaomei B, Fuli Z, Dan W. The application of VPN technology in the university's library [J]. Xi'an: 2011.

[16] Wenfeng Liu. The Application and Research of real-time database in air quality monitoring system [D]. Wuhan: Huazhong University of Science and Technology master's degree thesis, 2015. (In Chinese)

[17] Shuyi Yang, Zejun Wen, Sheng Jiang. Force control-based Remote Supervisory and Controlling System for Flow and Pressure of Water Supply [J]. Process Automation Instrumentation, 2007,28 ( 06 ) : 38-41. (In Chinese) 